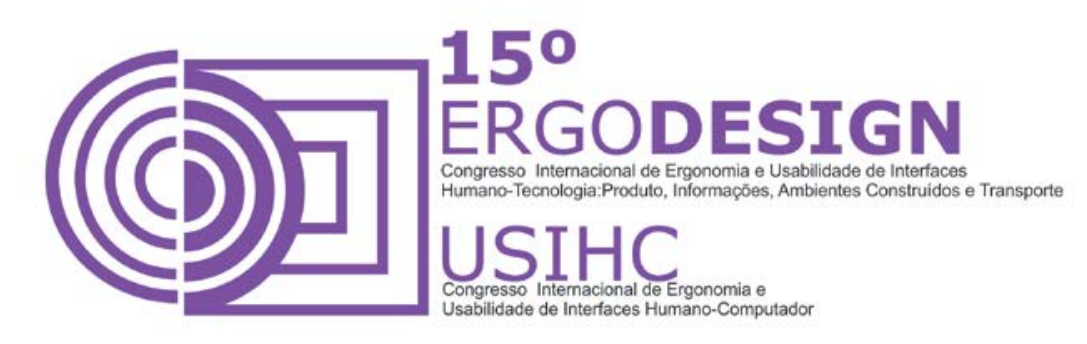

\title{
METODOLOGIA CENTRADA NOS PROTÓTIPOS: UM CAMINHO PARA INCLUSÃO DE USUÁRIOS NO PROCESSO DE DESIGN
}

\author{
ALCOFORADO, Manoel Guedes; \\ PASCHOARELLI, Luis Carlos; \\ SILVA, José Carlos Plácido da; \\ (1) UFPE, Doutor \\ E-mail: manoelguedes@hotmail.com.br \\ (2) UNESP, Doutor \\ E-mail: paschoarelli@faac.unesp.br \\ (3) UNESP, Doutor \\ e-mail:plácido@faac.unesp.br
}

\begin{abstract}
RESUMO
Diversos autores tem tratado sobre a complexidade da atividade projetual, apontando diversos fatores para a sua elevação, como: globalização, redução do ciclo de desenvolvimento e diversificação dos produtos, sustentabilidade, mudanças rápidas de comportamento dos usuários, novos materiais e tecnologias produtivas,... isso tem ampliado novamente as pesquisas sobre metodologia e estimulado 0 surgimento de novas tecnologias aplicadas ao desenvolvimento de projetos, como: digitalização 3D, tecnologias de prototipagem e manufatura rápida. Nesse contexto, após diversas pesquisas sobre metodologia e gestão do design, verificamos que o processo de design poderia se tornar mais interativo e eficiente se a atividade de prototipagem assumisse a função central do processo de design e se fossem usados os protótipos adequados a cada fase, estágio e propósito de design. Esses fatores poderiam melhorar o gerenciamento, a comunicação dentro da equipe e entre os demais atores do processo e ampliar a inserção e avaliação de requisitos dos usuários. Nesse sentido, este artigo apresentará os resultados da pesquisa de doutorado em design que propõe uma nova metodologia de projeto centrada no protótipo e os resultados obtidos após a realização de desafio projetual para experimentar e validação da referida metodologia.
\end{abstract}




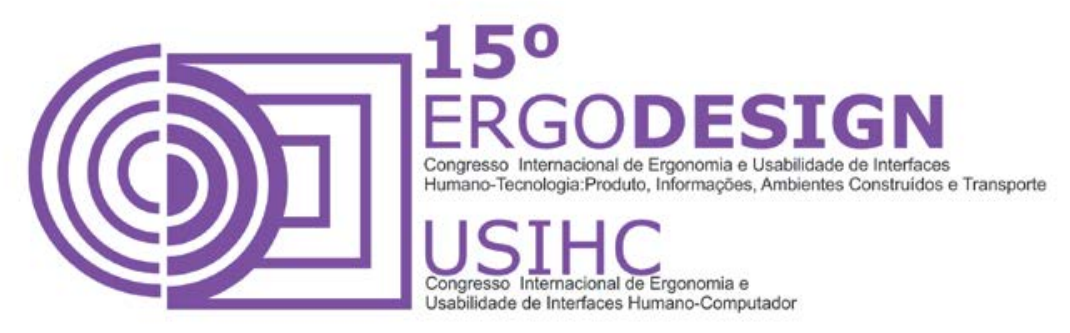

\begin{abstract}
Several authors have talked about the complexity of the design activity, pointing several factors to its elevation, such as: globalization, reduction of the development cycle and product diversification, sustainability, rapid changes in user behavior, new materials and production technologies, ... it has expanded the research in methodology and stimulated the emergence of new technologies applied to the development of projects such as: 3D scanning, rapid prototyping and rapid manufacturing technologies. In this context, after several research about methodology and design management, we find that the design process could become more interactive and efficient if the prototyping activity could assume the central role in the design process and if appropriate prototypes were used in each phase, stage and purpose of design. Those factors could improve the management, the communication inside the team and among other actors in the process and expand the insertion and evaluation of user requirements. Accordingly, this article will present the results of a doctoral research in design which proposes a new design methodology centered on the prototype and the results obtained after the completion of a projective challenge to experiment and validate that methodology.
\end{abstract}

\title{
1. INTRODUÇÃO
}

Temos definido o termo protótipo, como uma versão final do projeto ou produto que está sendo desenvolvido, em escala e materiais reais. Porém, segundo (ROGERS; SHARP; PREECE,2002) "os protótipos poderiam ser baseados tanto no desenho de uma tela, feito em papel, simulação de vídeo de uma tarefa, um mockup de papel ou cartão ou uma peça moldada em metal". Sendo assim, podemos definir o protótipo no sentido mais lato como sendo: "Artefato físico ou digital desenvolvido para compreender, explorar, avaliar e comunicar um ou mais atributos do produto que está sendo desenvolvido" (ALCOFORADO, 2007, p.39).

Nesse sentido, os protótipos poderiam ser realizados de forma horizontal, vertical ou de cenário (Greenberger,1998), com baixo, médio ou alto grau de fidelidade (Snyder, 2003; Ullman, 2003; Hold and Hill,2004; Rogers, Sharp e Preece, 2003; Righetti, 2005), e as diversas formas de representação dos protótipos dentro do processo de design, possibilitariam compreender os usuários e responder perguntas de forma concreta, materializando conceitos e tornando as características do projeto tangíveis.

Contudo, nas metodologias de design, observamos que os protótipos estão previstos apenas nas fases finais do projeto e que o seu uso está associado ao aumento do custo e tempo de desenvolvimento do projeto. Esses aspectos podem induzir os leitores a reduzir a produção e uso deles e a realizá-lo apenas nas fases finais do projeto.

Em oposição, acreditamos que ciclos iterativos, quando realizado com o protótipo adequado, tornam o desenvolvimento do produto mais eficiente e menos custosas, uma vez que, detectar e corrigir erros durante a fase de design é significativamente mais barato que detectá-las apenas na fase de produção. Nesse sentido, hoje, as organizações estão apostando no aumento quantitativo dos ciclos iterativos de desenvolvimento de protótipos, como forma de aumento qualitativo dos produtos. 


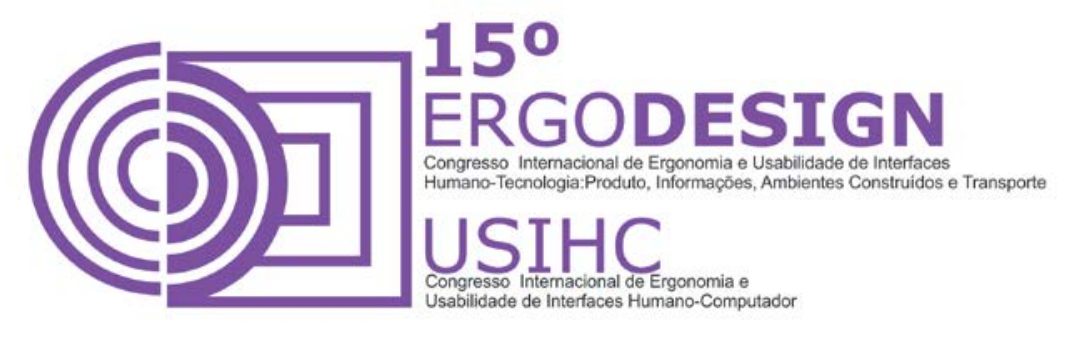

Sendo assim, diante das novas tecnologias de prototipagem, que permitem ampliar a sua utilização no design, esse artigo apresentará uma metodologia, que ao colocar o protótipo como ponto central do processo design, busca tornar o processo de design mais interativo, propondo um diálogo permanente de nossas ideias com o mundo real. O que pode ser feito através de versões iterativas do projeto, materializadas através dos protótipos. Para isso, apresentaremos o aplicativo desenvolvido para gerenciar o processo de design e indicar o protótipo, métodos e técnicas mais adequadas para cada etapa do projeto. Acreditamos que a metodologia apresentada pode contribuir para a criação de um processo mais fluído e estruturado, tornando mais eficiente o processo de design e os produtos gerados a partir dele.

\section{METODOLOGIA DE DESIGN}

Ao analisarmos a história de design, constatamos que o surgimento da metodologia de design, formalizada como temos hoje, possui relação direta com o aumento da complexidade projetual, com o contexto da época e com a cientificação do conhecimento adotado pela escola de Design, Hfg-Ulm na Alemanha (1952 à 1968).

Nessa época o design quanto prática de projeto havia se tornado uma atividade muito complexa. Dessa forma, precisava de uma teoria que correspondesse a sua complexidade. Complexidade essa formada não só pela constituição do objeto, pelo seu caráter interdisciplinar e transdisciplinar, mas, principalmente pela complexa multiplicidade do usuário.

Para Chistopher Alexandre (1964) considerado um dos pais da metodologia de design, a necessidade de sistematização e planejamento do processo de design se deve a diversos fatores dos quais podemos citar: (a) Complexidade - Os problemas de projeto se tornaram por demais complexos para que sejam tratados de forma intuitiva; (b) Quantidade de informações gerenciamento das informações envolvidas na solução de um problema. Dificuldade de coletar e manipular; (c) Quantidade de problemas - aumento significativo da quantidade de problemas envolvidos e (d) Espécies de problemas projetuais - Grande variação de problemas faz com que o design tenha dificuldade de experiências anteriores.

No período de 1958 à 1966 surgem as primeiras metodologias formalizadas e diversos métodos para aplicação do notável desenvolvimento do conhecimento científico da época no processo de design, entre os quais podemos citar: Horst Rittel (1958), Chistopher Jones (1984), Archer (1984), Gui Bonsiepe (1963) e Tomás Maldonado (1966).

Hoje, Vivemos em um contexto globalizado, onde a competitividade impulsiona cada vez mais as empresas locais a exercitarem práticas inovadoras como forma de adaptação, crescimento ou sobrevivência no mercado. Um cenário dinâmico, marcado pela aceleração do consumo e redução dos ciclos produtivos, as empresas precisam exercitar práticas inovadoras e investimentos em pesquisa e desenvolvimento, como estratégia de acompanhamento do mercado e dos seus concorrentes. Uma nova complexidade que vem justificando novamente a realização de pesquisas e o surgimento de novas metodologias e tecnologias de desenvolvimento de projeto. 


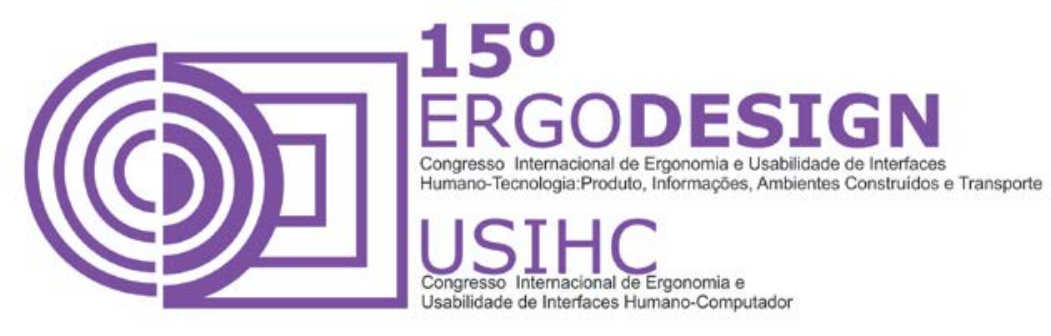

Para Cross (2005) ao analisar os diversos modelos de metodologia projetual apresentados por diversos autores afirma que há a necessidade de melhorar as formas tradicionais de projeto e uma preocupação de desenvolver novos procedimentos. Uma das justificativas seria o aumento de complexidade nos produtos modernos com uma grande variedade de novas demandas e de produtos que nunca existiram antes. Sendo assim, "uma abordagem nova e mais sistemática é necessário" (CROSS, 2005, p.45).

A análise desses aspectos apresentados sobre a redução do ciclo de desenvolvimento do produto amplia a importância das pesquisas nas áreas de metodologia, gestão de desenvolvimento de produto, de métodos, técnicas e ferramentas que possam contribuir para um processo de design mais eficiente, de forma a atender essas demandas atuais de mercado e da indústria.

\section{A IMPORTÂNCIA DOS PROTÓTIPOS NO PROCESSO DE DESIGN}

Estudos realizados revelam a importância das representações como estratégia para ampliação de nossas capacidades mentais e habilidades cognitivas (Ullman et al 1990; Norman, 1988; Rogers, Sharp e Preece, 2006). Dessa forma, a elaboração de desenhos, esboços, diagramas, mapas, notas, modelos etc. teriam uma função maior que puramente representar nossas ideias, elas permitiriam "externalizar" dados que dificilmente poderiam ser analisados apenas através de processos mentais, ou seja, no caso do design, contribuem para ampliarmos nossa capacidade cognitiva e no caso, projetuais.

"O ambiente externo - papel e lápis, computadores, livros - possui um número de funções no processo de design: é a fonte de informação, é uma capacidade analítica, é um facilitador de documentação e comunicação, e o mais importante para os designers, é uma extensão para a memória de curto prazo". (ULLMAN, 2010, p.55)

Dessa forma, acreditamos que o uso do protótipo dentro do processo de design dá ao design a possibilidade de responder perguntas de forma concreta, materializando conceitos e tornando características tangíveis. Com ele, o designer pode obter informações do contexto e explorar ideias através da produção de artefatos comunicativos e interativos.

"Eu acredito que se nos pensarmos primeiro sobre as pessoas e então testar, testar e testar novamente os protótipos de nosso design, nós teremos uma boa chance de criar soluções inovadoras que as pessoas irão valorizar e apreciar". Moggridge (2006).

Jones e Marsden (2006) apontam 3 atividades que devemos explorar para trabalhar de forma efetiva com design interativo dentro do processo de design: (1) Compreender usuários: suas capacidades e limitações, detalhes de como vivem, o que fazem e usam (2) Desenvolver protótipos de design: criar representações do design para promover interações que possam demonstrar, alterar e discutir o design (3) Avaliação: cada protótipo é um estágio melhorado e refinado do anterior que evolui através de técnicas de avaliação que identifiquem pontos fortes e fracos do design e principalmente dar o poder e segurança a equipe de tomar decisões sobre a continuidade e descontinuidade do design. 

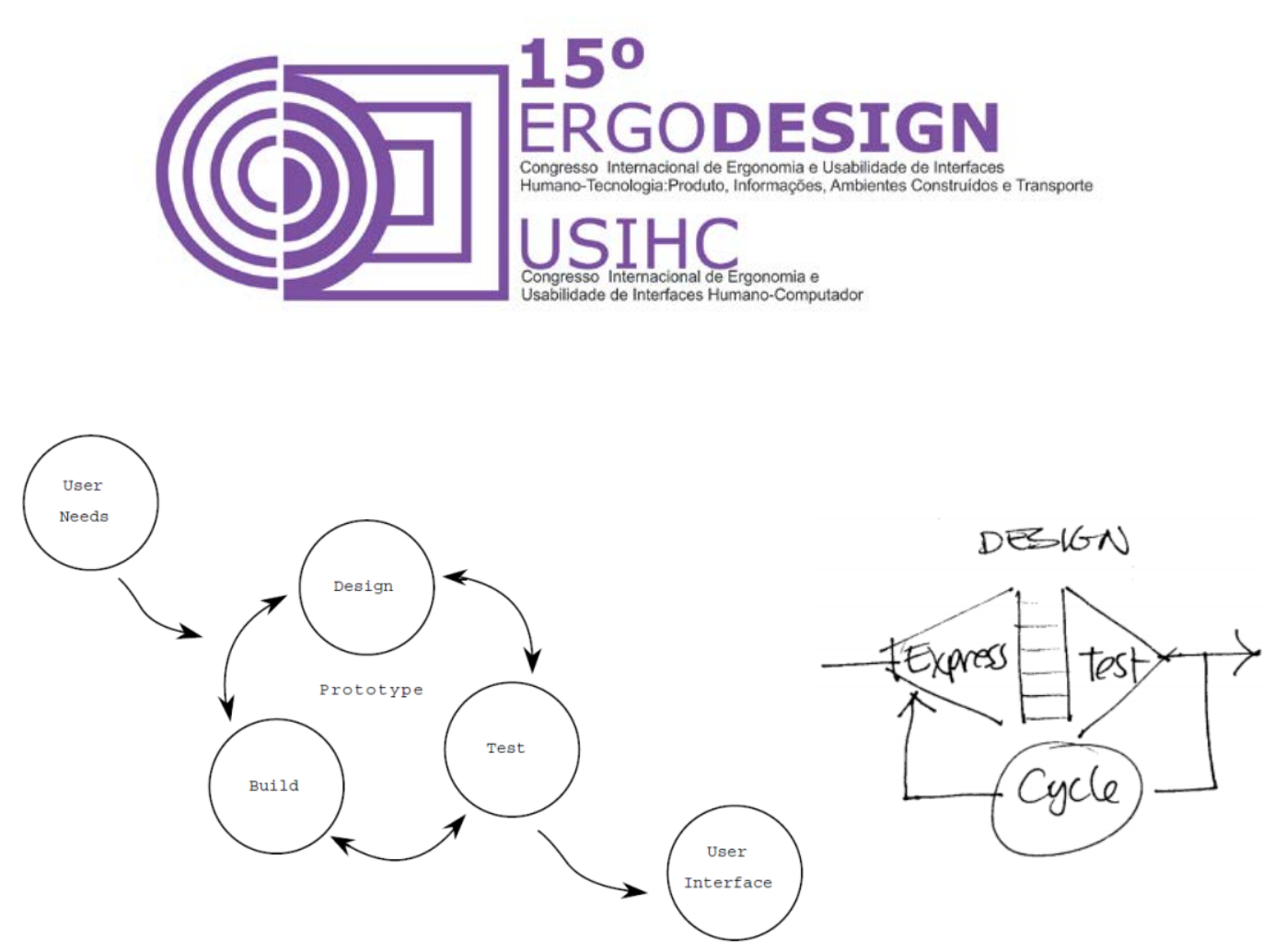

Figura 1 - Modelo de processo de design de Gayle Curtis e Vertelney (1990, p.07) e Figura 2 - Esquema de ciclo iterativo de expressão de idéias e testes proposto por Bill Verplank (2009, p.04).

Dessa forma, podemos entender que a essência do processo de design pode ser concentrar em 3 atividades: Projetar, Construir e Avaliar. Nelas o protótipo é como peça fundamental do processo iterativo. $\mathrm{E}$ isso pode e deve ser feitos em ciclos iterativos controlados e sistematizados, onde se possa medir e comparar os resultados com ciclos anteriores, de forma a ter elementos suficientes para um processo de tomada de decisões confiáveis ao longo de todo processo de design.

\section{METODOLOGIA DE DESIGN, CONCEITO E USO DE PROTÓTIPOS}

Epistemologicamente, protótipo seria a materialização do conhecimento. Literalmente significa: "o primeiro de um tipo". Segundo Baxter (1998), no processo de design de produtos, a palavra se refere aos dois tipos de representação: (1) no sentido mais preciso, refere-se a representação física do produto que será produzido industrialmente, (2) no sentido mais lato, refere-se a qualquer tipo de representação física construída com o objetivo de realizar testes físicos.

O termo prototyping no dicionário tem sido definido como: "criação de um modelo, ato de construir um exemplar original", relatando o uso de modelos ideais de exemplo ou demonstração realizado através da construção de pequenas partes de alguma coisa, como amostra para determinar a qualidade de alguma coisa, através de testes ou simulação realizados em um exemplar. Dessa forma, o protótipo não está limitado a algo físico, nem a representação de todas as características de um produto.

Genericamente diversos autores em todo o mundo se dirigem as etapas de representação de características de um produto ou sistema através do termo prototipagem (prototyping), dividindo-os de acordo com o nível de fidelidade (baixa e alta fidelidade). Dessa forma: sketches, mockups, modelos e protótipos tradicionais, são tratados como tipos de protótipos com diferentes níveis de fidelidade. 


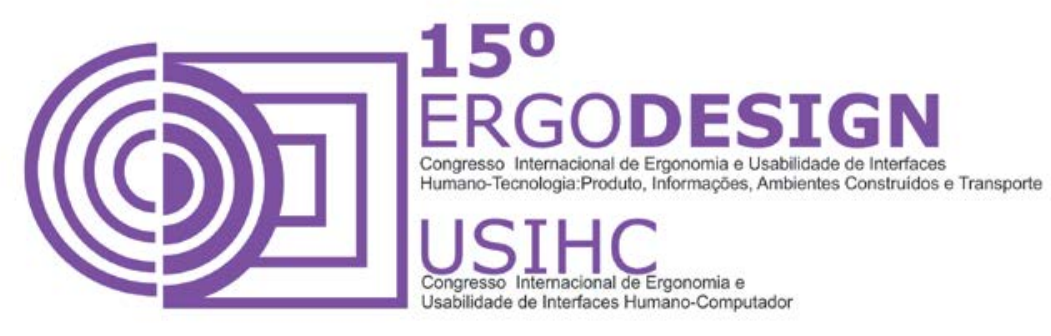

Dessa forma, considerando as áreas de design de produto, gráfico e digital, temos usado a definição apresentada para protótipos: "um artefato físico ou digital desenvolvido para compreender, explorar, avaliar e comunicar um ou mais atributos do produto que está sendo desenvolvido" (ALCOFORADO, 2007, p.39).

\subsubsection{Classificando os protótipos}

Buchenau e Suri (2005) descreve que protótipo são desenvolvidos com três finalidades: (1) estabelecer uma compreensão da experiência do usuário ou do contexto, (2) explorar e Avaliar as idéias de design e (3) comunicar essas idéias a uma audiência, e em 3 níveis: "look like"(visual), "Behaves Like"(comportamento) e "Work Likes" (funcionamento).

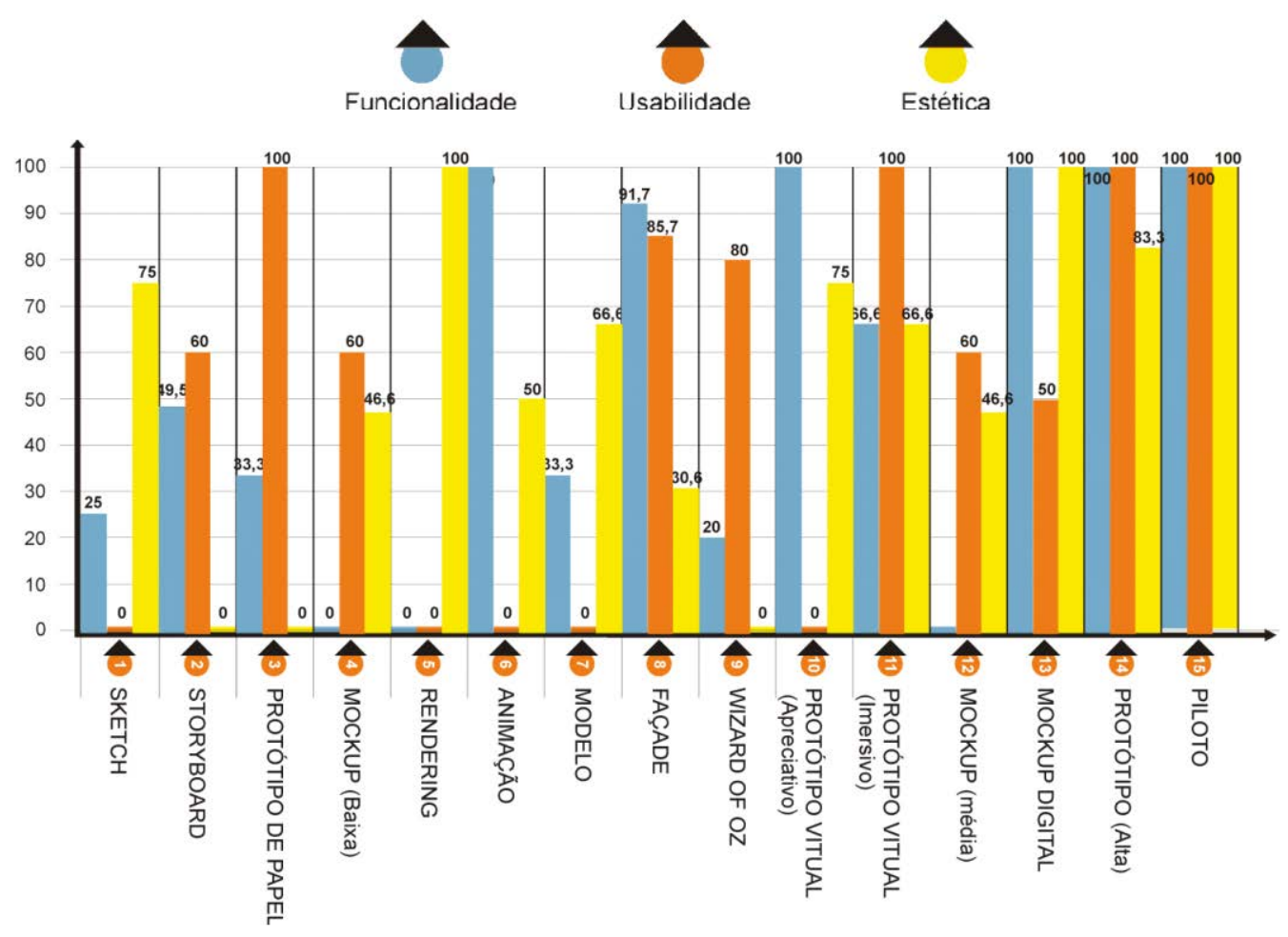

Figura 2 - Quadro geral do potencial de comunicação dos protótipos (fonte: autor)

A partir dessas classificações percebemos que os protótipos são utilizados para avaliação de 3 níveis de interação com os produtos: (a) funcionalidade, (b) usabilidade e (e) estética.

Com objetivo de verificar e classificar a aplicação dos protótipos na realização de avaliações estéticas, funcionais ou de usabilidade do Design, consultamos diversos estudos da área e 


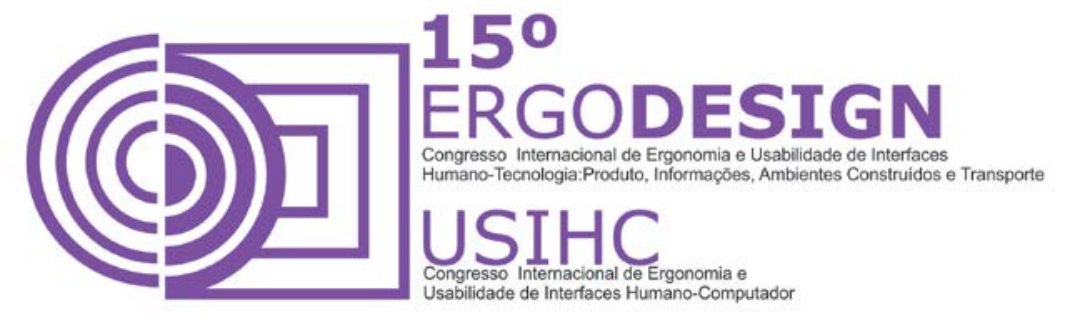

realizamos recortes nas definições e aplicações de cada um dos tipos de protótipos descritas por diversos autores, procurando identificar a aplicação dos protótipos estudados para cada uma dos canais de comunicação de: funcionalidade, usabilidade e estética. Com tratamento estatístico de média proporcional chegamos ao gráfico apresentado na figura 2 onde é possível classificar os protótipos nesses 3 canais.

Os protótipos também podem ser classificados pelo nível de fidelidade, em (a) baixa, (b) média e (c) alta fidelidade ((Snyder, 2003; Ullman, 2003; Hold and Hill, 2004; Preece et al, 2006; Righetti , 2005. Sendo assim, poderíamos classificar os protótipos por fidelidade segundo a figura 3.

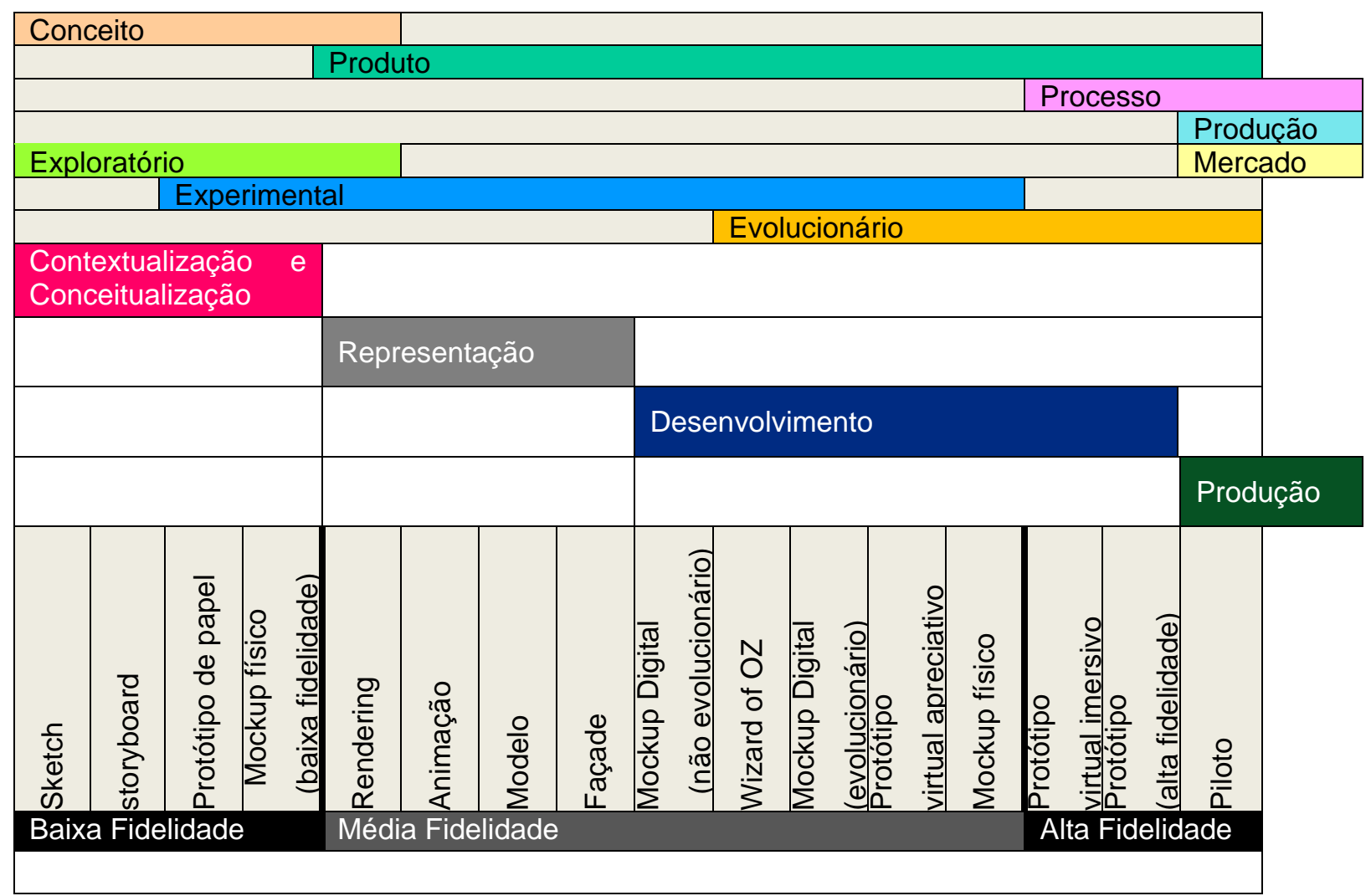

Figura 3 - Painel completo das relações da finalidade, áreas, estágios e propósitos com os tipos de protótipos (fonte: Autor)

Classificamos os protótipos também por sua finalidade de uso no processo de design, em: (I) Contextualização e Conceitualização, (II) Representação, (III) Desenvolvimento e (IV) Produção. Eles podem ser definidos da seguinte forma: (I) Permite contextualizar o problema, o cenário, o mercado, o cliente ou produto, ou ainda, definir conceitos preliminares do artefato que será proposto para eles. (II) tem por objetivo apresentar uma proposta de projeto para os demais atores do processo de design, (II) Tem por objetivo desenvolver um conceito gerado, 


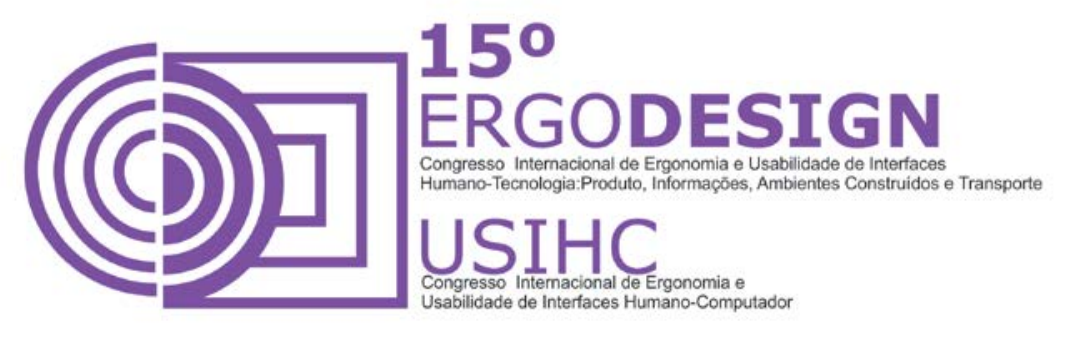

podendo evoluir até a proposta final e (IV) Produção - Tem por objetivo testar os aspectos produtivos da proposta final, avaliando a relação da proposta final com as tecnologias produtivas, qualidade, sustentabilidade, viabilidade e seriabilidade.

Ullman (2010, p.117), que propõe e descreve quatro classes de protótipos: protótipo de conceito, de produto, de processo e de fabricação, baseados na função e estágio de desenvolvimento do produto. Acrescentaremos a essa classificação um novo item classificatório, o protótipo de mercado. Baseados nisso poderíamos classificar os protótipos conforme a figura 3.

Budde et al (1992) define que protótipos podem ser caracterizados através de estágios de desenvolvimento. Cada estágio auxilia o designer a esclarecer os requerimentos definidos do projeto. Ele define 3 estágios dos protótipos: (a) Exploratório, (b) Experimental e (c) evolucionário. (a) Exploratório - Deve ser usado quando o problema não está claro suficiente para definirmos caminhos para o desenvolvimento de soluções projetuais, requerimentos do futuro produto e sistema ou até mesmo para reconhecer o verdadeiro problema projetual; (b) Experimental - Deve ser usado para implementação técnica do produto, ou seja, incluindo questões de ordem funcional ou ergonômica do produto e (c) Evolucionário - permite realizar o acompanhamento do desenvolvimento do produto ou sistema até o produto final, ou seja, através de ciclos iterativos contínuos controlados e avaliados até o produto alcançar o nível de evolução adequada. Os protótipos em relação aos estágio podem ser classificados conforme figura 3.

\section{METODOLOGIA DE DESIGN MEDIADA POR PROTÓTIPOS}

\subsection{Construção da metodologia}

A partir das macrofases: Preparação, Desenvolvimento e Realização, definidas pela síntese das metodologias de Assimov (1962), Gui Bonsipe (1978), Archer (1984), Loback (2001), Munari (2002), Cross (2005), Rozenfeld (2007), Baxter (2008) e Ulman (2010) e das microfases definidas pela nossa fundamentação teórica de nosso estudo que coloca o protótipo, as etapas de design, construção e avaliação e ainda os ciclos iterativos, no centro do processo de design, desenvolvemos uma estrutura básica para a metodologia centrada em protótipos (figura 4). Nela definimos portões de aprovação na passagem de uma Microfase ou Macrofase para outra, utilizando como referência os estudos de Engenharia Simultânea (total design) de Purg, (1990), do funil de desenvolvimento de Clark e Wheelwright (1994), Phase Review de McGrath (1992), Stage-Gates de Robert G. Cooper (2011) e Modelo de maturidade de modelo da maturidade (CMMI do SEI) e (OPM3 da PMI). 

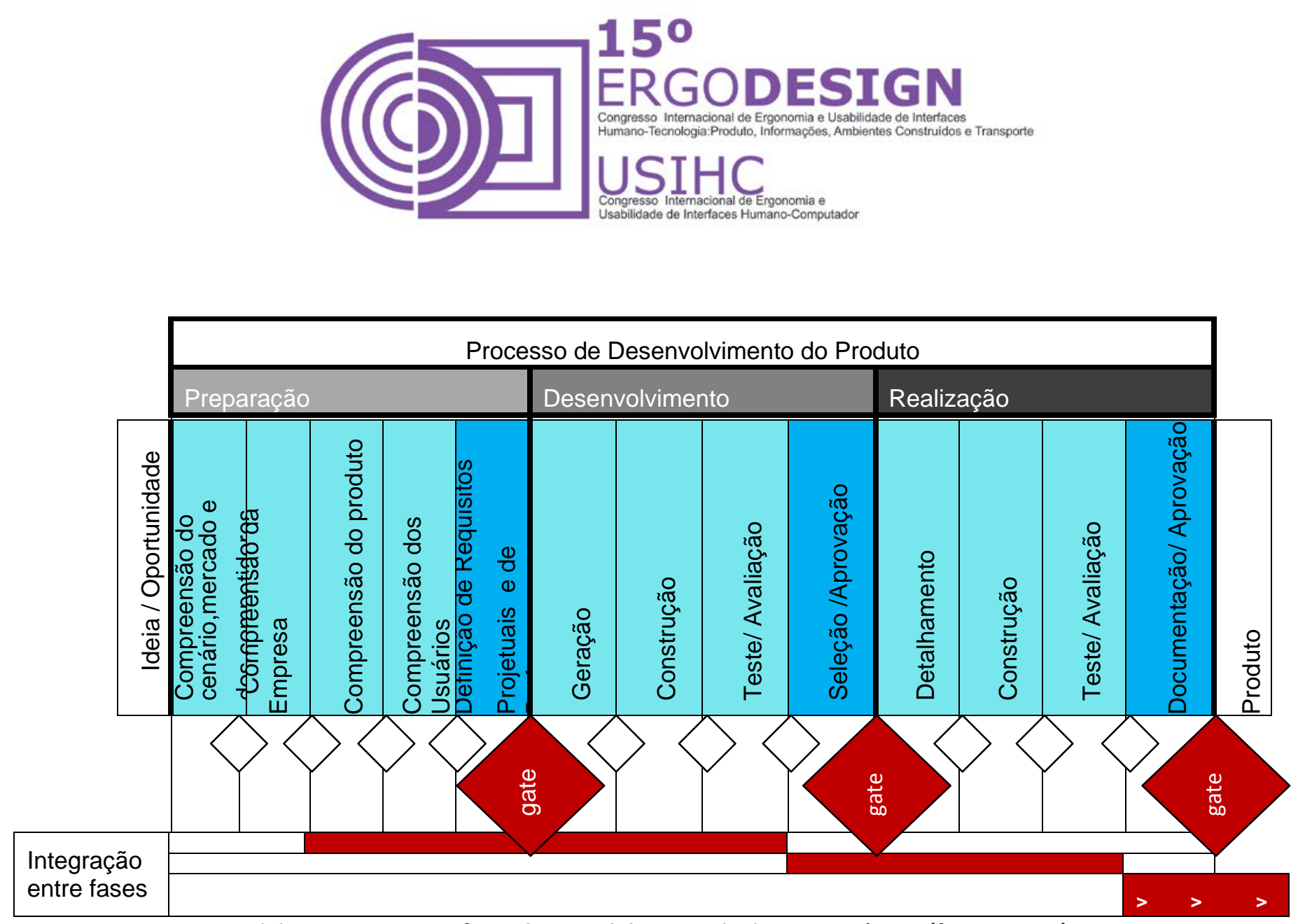

Figura 4 - Painel das macro e micro fases da metodologia mediada por protótipos (fonte: Autor)

Nele, deveria ser avaliado as atividades previstas para aquele estágio, os atendimento aos requisitos definidos na fase inicial, as alternativas que tiveram o melhor desempenho e a maturidade da solução final para uma tomada de decisão com menor incerteza. Esses portões podem gerar aprovação ou retorno a fases anteriores, para geração de novos ciclos de desenvolvimento.

\subsection{Ferramentas da metodologia}

Com base na metodologia criada e nos resultados de nosso estudo, desenvolvemos duas ferramentas de auxílio a aplicação da metodologia, seleção de protótipos e realização de avaliações para auxiliar os design no processo de design centrado em protótipos: (a) Aplicativo de seleção e avaliação de protótipos, (b) Planilha de Registro dos requisitos, pesos, metas, resultados da avaliação e tomada de decisões e (c) Cartões de métodos e técnicas para orientar o uso dos protótipos, apresentados na figura 5. 

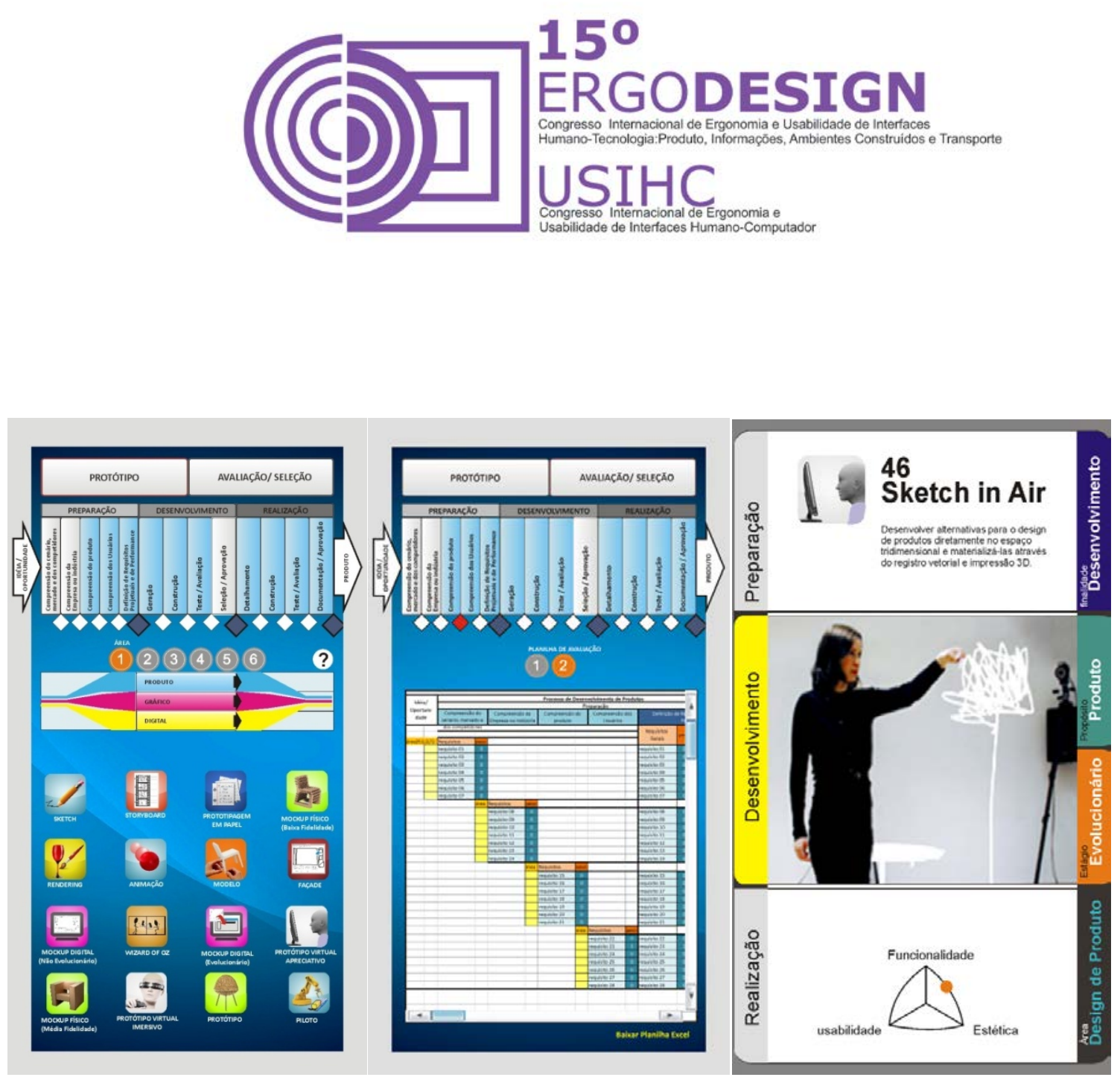

Figura 5 - Aplicativo para orientar a seleção e avaliação de protótipos, planilha de registro e acompanhamento do processo de design e Cartões de métodos de prototipagem (fonte: Autor)

\subsection{Experimento}

Visando avaliar os resultados da aplicação da metodologia centrada em protótipos, estruturamos um desafio projetual entre duas equipes de alunos do curso de design da Universidade Estadual Paulista (UNESP), situada em Bauru, São Paulo, Brazil. A equipe experimental foi capacitada com a metodologia proposta e a outra, de controle, utilizou a metodologia ensinada e praticada na UNESP, baseada em alguns dos autores pesquisados.

O projeto do experimento foi submetido ao Comitê Nacional de Ética em Pesquisa CONEP, em Março de 2013, através da Plataforma Brasil, sob o número de processo 14906713.2.0000.5398, tendo sido aprovado tanto pelo parecer consubstanciado do CEP da Faculdade de Ciências da UNESP em 24/04/2013 (parecer 323.380). Todos os alunos que participaram do experimento assinaram o termo de consentimento livre e esclarecido que dava ciência dos objetivos e termos do experimento.

O desafio teve a duração de $8 \mathrm{~h}$, divididos em 2 períodos de $4 \mathrm{~h}$. As equipes eram formadas por 6 alunos cada e desenvolveram as atividades em dias consecutivos, sendo disponibilizado a ambas equipes a mesma estrutura: dois laboratórios da UNESP, um para modelagem 


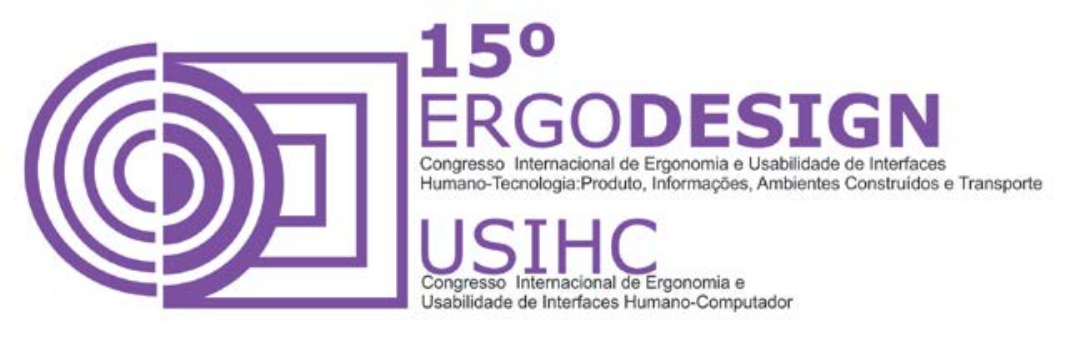

tradicional de modelos e protótipos e outro para uso de novas tecnologias de prototipagem: Prototipagem virtual e rápida, com computadores, softwares, impressoras 3D, Fresadoras e routers CNC, todo material de desenho e modelagem além de espaços para reuniões e desenvolvimento e avaliação de alternativas.

O Briefing propunha o desenvolvimento de um relógio celular inovador para empresa Swatch de relógios, adequado às necessidade do cenário, mercado, empresa e usuários.

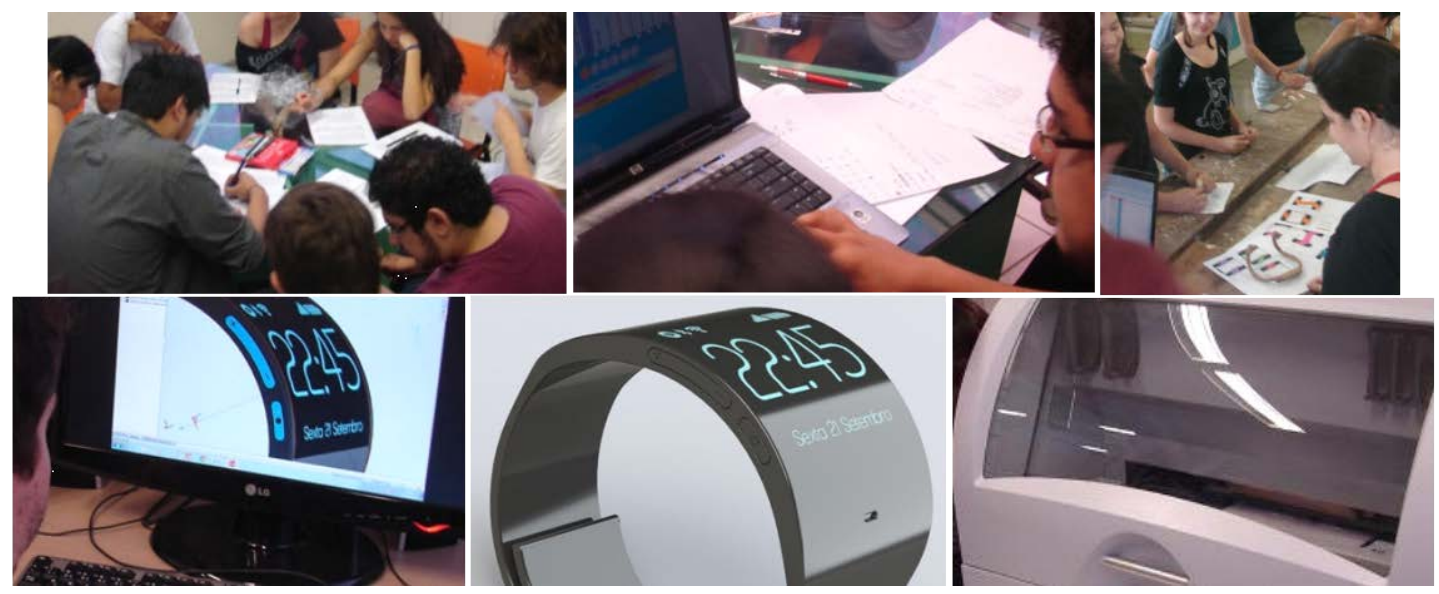

Figura 6 - Equipe experimental desenvolvendo o projeto durante o desafio projetual (fonte: Autor)

A proposta era que eles integrassem em um só artefato o conhecimento de desenvolvimento do design de produtos, gráfico e de interfaces. Além das orientações do briefing era apresentado as equipes usuários com o mesmo perfil do briefing, caso elas desejassem realizar coleta de informações ou avaliações com as alternativas e produtos gerados. Isso foi importante para avaliar a inclusão de usuários em ambas as metodologias e processos realizados pelas equipes.

A coleta de dados do experimento ocorria: (a) através de registro de áudio e vídeo, através de câmeras de segurança instaladas em todos os ambientes utilizados e do software D-view Cam da empresa D-link, (b) do registro manual das atividades em intervalos de 5 em 5 minutos realizada por 3 monitores e (c) através do registro fotográfico com sincronização de data e horário. 

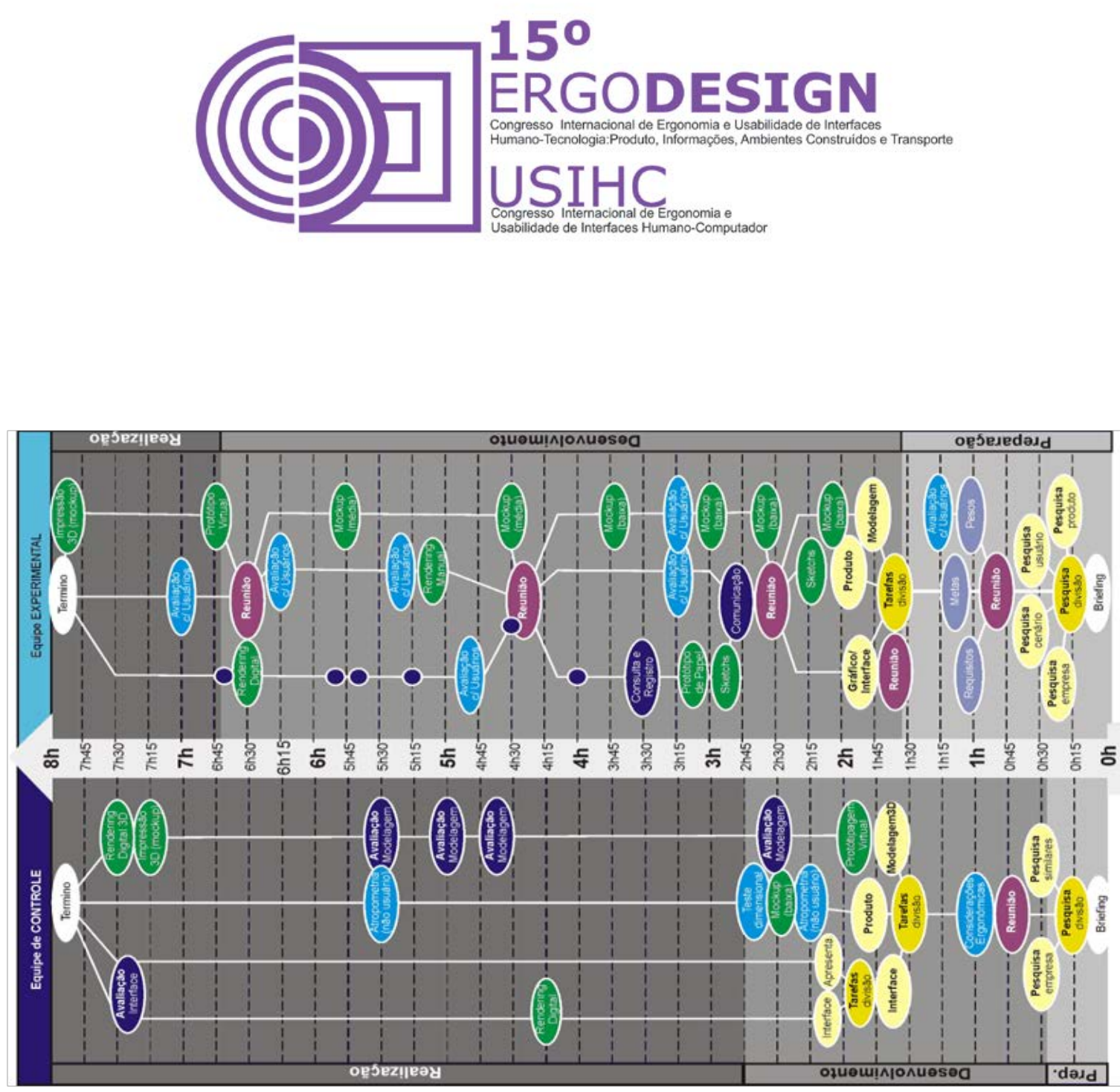

Figura 7 - Comparativo das sínteses dos fluxogramas gerais de atividades desenvolvidas pelas equipes de controle e experimental. (fonte: Autor)

Após a coleta de dados, foram sincronizadas todas as informações coletadas e realizada uma análise do processo de design desenvolvido por ambas as equipes utilizando 10 indicadores previamente definidos e justificados em nossa pesquisa teórica. Os indicadores foram: (1) Indicador do acompanhamento periódico do PDP; (2) Indicador da inclusão e avaliação de parâmetros do usuário e do cenário, (3) Indicador do uso e adequação dos Protótipos às fases do PDP, (4) Indicador da divisão de problemas em sub-problemas. (5) Indicador da continuidade do fluxo de atividades nas fases do PDP, (6) Indicador dos custos no PDP, (7) Indicador da qualidade de comunicação, da gestão da informação e tomada de decisão no PDP, (8) Indicador do tempo nas fases do processo de design, (9) Indicador da qualidade e viabilidade do produto e (10) Indicador da integração das fases do processo de design.

O para estruturação dos dados do experimento utilizou a metodologia usada por Griffiths (2004) e Gill (2005) que permitiram gerar diversos gráficos para análise do fluxo de atividade e registro de cada ocorrência dos indicadores. Entre os gráficos gerados está o da síntese geral das atividades desenvolvidas pelas equipes (figura 11). 


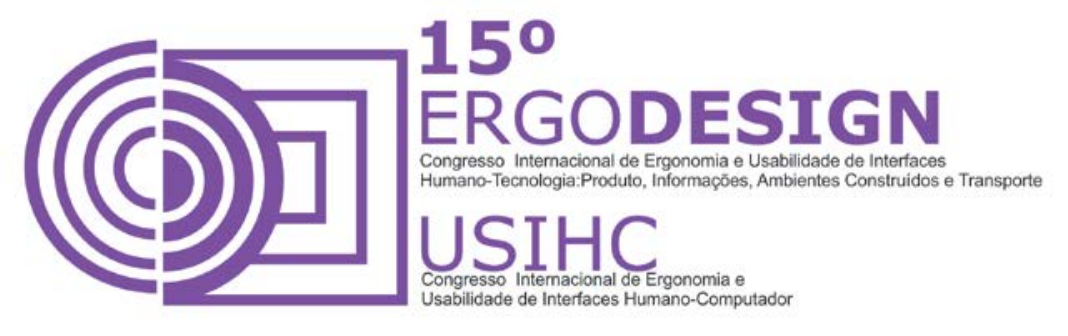

A análise foi realizada a partir dos gráficos estatísticos comparativos dos dados quatitativos obtidos. Esses registros quantitativos, eram gerados a partir do registro de ocorrências desses indicadores ao longo do desenvolvimento das atividades de cada equipe. Contudo, foi realizada também uma análise qualitativa a partir dos registros dos monitores e dos dados do sistema de áudio e video, que permitiram complementa e aprofundar a análise dos diálogos e atividades desenvolvidas por essas equipes.

\subsection{Experimento}

A equipe experimental, ao utilizar a metodologia e as ferramentas propostas por essa, conseguem obter resultados expressivos no: (a) Gerenciamento de encontros para acompanhando periódico do PDP ao longo de todo desenvolvimento do projeto; (b) Realização de inclusões de parâmetros do cenário e dos usuários, principalmente nas atividades de: geração de requisitos projetuais, definição de conceitos do projeto, realização de avaliações com a participação desses usuários e aprovação do produto final; (c) Utilização de um número maior de protótipos ao longo do processo, desde o início do projeto e de forma adequada a cada fase, estágio e propósito de design; (d) No processo de comunicação entre os membros e entre os usuários, gestão da informação dos requisitos e avaliações e tomada de decisão, com uso da planilha de acompanhamento, ao longo de todo desafio.

Figura 13 - Gráfico estatístico comparativo de todas as porcentagens dos indicadores (fonte: autor)

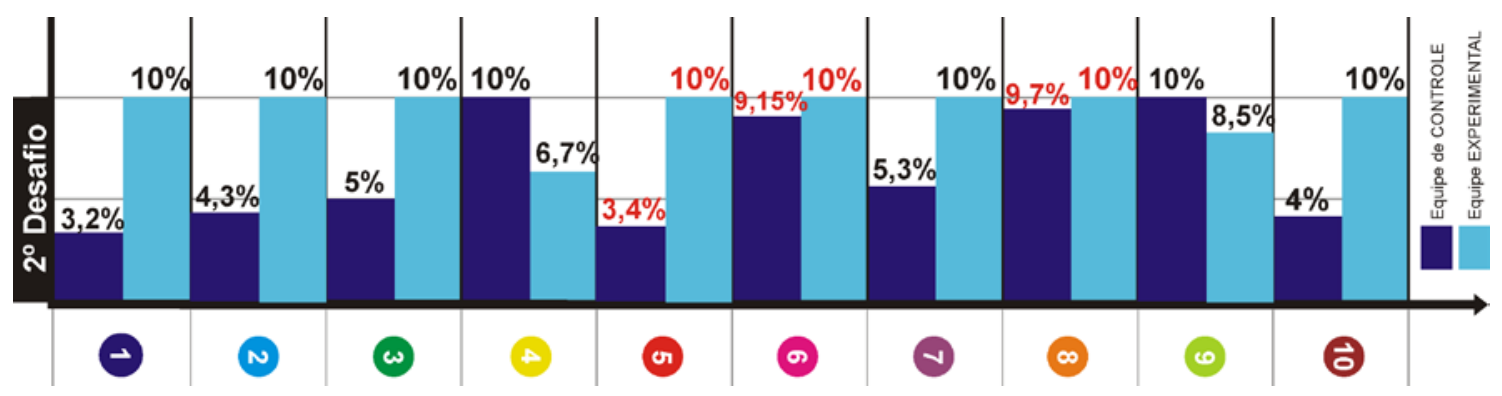

O gráfico 13 apresenta o resultado dos 10 indicadores pesquisados. Nele observamos de fato que a equipe experimental consegue resultados expressivos nos itens: (1) acompanhamento periódico do PDP; (2) Inclusão e avaliação de parâmetro do usuário e do cenário; (3) Uso e adequação dos protótipos às fases do PDP; (5) indicador de continuidade do fluxo de atividade do PDP; (7) Indicador de comunicação, Informação, Gestão e tomada de decisão e (10) Integração entre as fases do PDP.

A partir do gráfico podemos afirmar que a equipe experimental, na média total dos resultados, foi $31,15 \%$ mais eficiente no processo metodológico de design que a equipe de controle, 


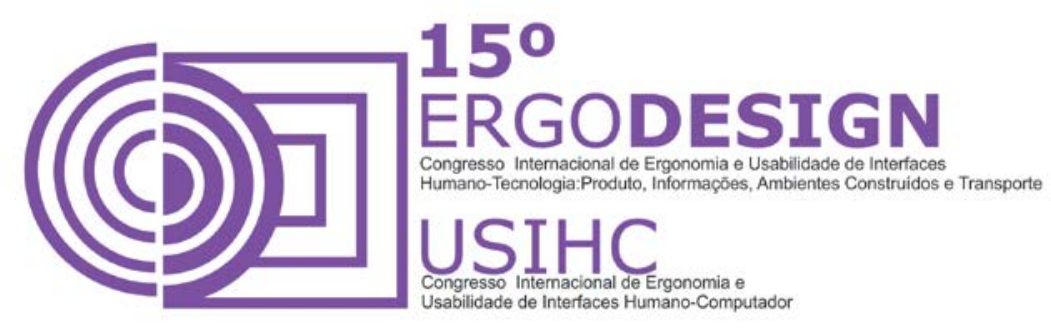

fazendo com que os resultados gerais apresentam uma diferença expressiva de desempenho a favor da equipe experimental, que aplicou a metodologia proposta pelo nosso estudo.

\section{CONCLUSÃO}

A metodologia proposta se mostrou bastante eficiente em aspectos importantes da atividade projetual : (a) inclusão dos usuários no processo de design, tanto nas fases iniciais, para geração de requisitos, pesos e metas para o projeto, como para definição de conceitos, parâmetros e realização de avaliações das alternativas e dos produtos gerados; (b) Uso e adequação dos protótipos, de baixa, média e alta fidelidade, ao longo de todo processo de design, contribuindo para melhorar a comunicação entre os membros da equipe, a compreensão das necessidades dos usuários a das alternativas geradas, bem como a realização de um número maior de avaliações que permitiram identificar e selecionar de forma mais precisa as melhores alternativas; (c) Gerenciamento do processo de design e de tomada de decisões, permitindo gerenciar de forma periódica as etapas, registrar os requisitos, pesos e metas, e ainda, acompanhar os resultados das avaliações de forma identificar as melhores alternativas, a necessidade de gerar novos ciclos iterativos com uso de protótipos e o momento adequado para término do projeto e aprovação do produto final, a partir das metas e níveis de maturidade estabelecidos.

Acreditamos nos princípios que embasaram a metodologia centrada em protótipos: (a) Tornar o processo de design mais interativo, ampliando o uso de protótipos para todas as fases do processo de design; (b) Realização de um número maior de ciclos iterativos com uso do protótipo adequado, considerando as características das fases, área de design, finalidades, estágio e propósitos; (c) compreendendo a relação desses protótipos com tempo e o custo envolvidos por cada uma das tecnologias de prototipagem, adequando o nível de fidelidade a cada grau de incerteza do processo, ampliando as apostas a medida que essa incerteza se reduz ao longo das fases e (d) Ampliação da visão dos protótipos para algo além de uma ferramenta de apresentação do projetos, compreendendo que elas podem contribuir desde a compreensão do problema e do que deve ser projetado, nas fases iniciais de design, como para levantamento de parâmetros com usuários, realização de avaliações com as alternativas e aprovação do produto final. Acreditamos que esses princípios podem contribuir para tornar o processo de design e os produtos desenvolvidos a partir dele mais eficiente, por acreditar que da mesma forma que uma criança precisa interagir com o mundo para se desenvolver melhor, um produto precisa de versões interativas, ou seja, de protótipos para poder evoluir como desejamos.

\section{REFERÊNCIAS BIBLIOGRÁFICAS}

Alcoforado, Manoel Guedes. Comunicação intermediada por protótipos. Tese de Mestrado. UFPE, 2007.

Arleth, Jens. Streamlining Your Company's Development Process: Opportunities made available by introducing Stage-Gate. Denmark: Stage-Gate.EU, 2011. Disponível em: http://www.stage- 


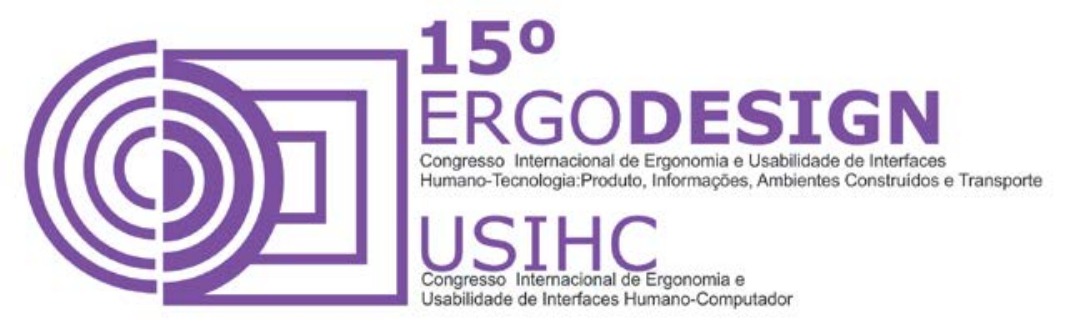

gate.eu/articles Acessado em 14/05/2013.

Asimov, Morris. Introduction to Design. Prentice-Hall, New Jersey, 1962

Baxter, Mike. Projeto do produto: Guia Prático para o desenvolvimento de novos produtos. Tradução de Itiro lida. São Paulo: Edgard Blucher,1998.

Bonsiepe, Gui. Teoria y práctica del diseño industrial. Barcelona: Gustavo Gli, 1978.

Buchenau, Marion; SURI, Jane Fulton. Experience Prototyping. Proceedings of the conference on Designing interactive systems: processes, practices, methods, and techniques. New York, p.424433, 2000.

Budde, R. Kautz, K; Kuhlenkamp, K; H.Zullighoven Prototyping: an approach to evolutionary system development. Berlin: Springer, 1992.

Clark, K. B. Wheelwright, S. C. Managing New Product and Process Development. New York: The Free Press, 1993. 896p

Cross, Nigel. Engineering Design Methods: Strategies for Product Design. 3 Ed. John Chichester: Wiley \& Sons Ltd, 2005.

Degraff, Jeff. Lawrence, Katherine A. Creativity at Work: Development right Practices to make innovation happen. San Francisco: Jossey-Bass, 2002.

Gayle Curtis, Laurie Vertelney. Storyboards and Sketch Prototypes for Rapid Interface Visualization, CHI 1990 Tutorials.

Greenberger, Saul. Prototyping for Design and Evaluation.CPSC. California, 1998.

Hartmann, Bjorn. S. R. Klemmer et al. Reflective physical prototyping through integrated design, test, and analysis. In Proceedings of UIST 2006: ACM Symposium on User Interface Software and Technology. Montreux, Switzerland, 2006.

Houde, Stephanie. Hill, Charles. What do Prototypes Prototype? Cupertino: Apple Computer, Inc, 2004.

Jones, Matt. Marsden, Gary. Mobile Interaction design. Chinchester: John Wiley \& Sons Ltd, 2006.

Lobach, Bernd. Design Industrial: Bases para configuração dos produtos industriais. Tradução de Freddy Van Camp. São Paulo: Edgard Blucher, 2001.

Moggridge, Bill. Designing Interactions. Mit Press, 2006.

Moraes, Dijon de. Metaprojeto: o Design do Design. São Paulo: Edgard Blucher, 2010.

Munari, Bruno. Das coisas nascem as coisas. São Paulo: Martins Fontes, 2002. 


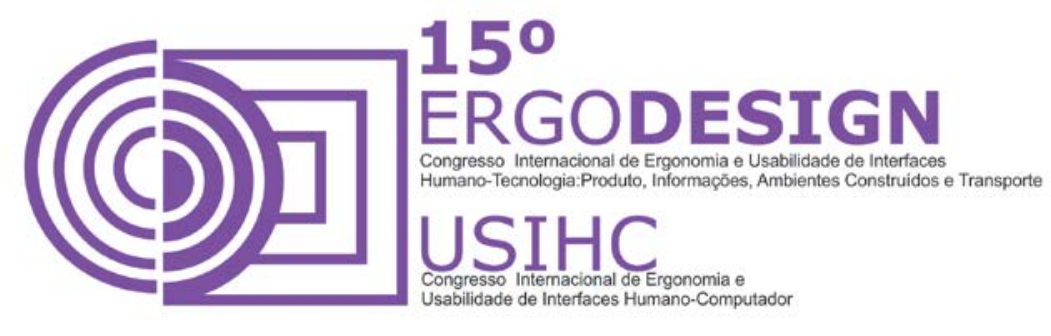

Newell, A. Simon, H.A. Human Problem Solving. Englewood Cliffs N.J: Prentice Hall, 1972.

Norman, Donald A. The Psychology of Everyday Things. USA.: Basic Books, 1988.

Preece, Jenny. Rogers, Yvonne and Sharp, Helen. Interaction Design: Beyond Human-computer Interaction. New Delphi: Wiley India, 2006.

Purg, Stuart. Total Design. Addison-Wesley, New York, 1990

Righetti, Xavier. Study of prototyping tools for user interface design. Tese de doutorado. Geneva: University of Geneva, 2005.

Rozenfeld, Henrique et al. Gestão de Desenvolvimento de Produtos: uma referência para melhoria do processo. São Paulo: Saraiva, 2006.

Snyder, Carolyn. Paper Prototyping: The fast and easy way to define user interfaces. San Francisco: Morgan Kaufmann Publishers, 2003.

Ullman, David G. The Mechanical Design Process. 4th edition. New York: Mc Graw Hill, 2010.

Ullman, D. G. The mechanical design process. 3a Edição. New York : McGraw-Hill,2003.

Ullman, D. G, Wood, Stephen, Craig, David. The Importance of drawing in the mechanical design process. Computers \& Graphics. v. 14, n.2, p.263-274, 1990.

Verplank, Bill. Interaction Design Sketchbook. 15 July 2009. disponível em: http://hci.rwthaachen.de/tiki-download wiki attachment.php?attld=797 acessado em 10/11/2013. 